\title{
Abrupt decrease of c-myc expression by antisense tran- scripts induces terminal differentiation and apoptosis in human promyelocytic leukemia HL-60 cells ${ }^{1}$
}

\author{
HaO Xiu Juan, Pei Hien TANG ${ }^{2}$, Xiu Sen Li, Fei \\ Zi JiANG, Yong Zhi Xi, Ning MAO, De Lin DU, \\ Min WU* \\ Institute of Basic Medical Sciences, Beijing 100850 \\ * National Laboratory of Molecular Oncology, In- \\ stitute of Cancer Research Beijing, Chinese \\ Academy of Medical Sciences, Beijing 100021
}

\section{ABSTRACT}

This study was designed using c-myc antisense transcripts to evaluate how alteration of c-myc expression in human myeloid leukemic HL-60 cells could influence the myelomonocytic differentiation and induction of apoptosis. The recombinant plasmid pDACx expressing antisense transcripts to c-myc fragment containing a part of intron 1 and $137 \mathrm{nt}$ exon 2 was constructed, pDACx was transfected into HL-60 cell line by lipofectin reagent. Cytochemical stainings including NBT reduction, peroxidase and $a$-NAE as well as detection of CD13 and CD33 antigens by flow cytometric analysis indicated occurrence of myelomonocytic differentiation in cells expressing antisense transcripts to c-myc. DNA degradation measured by DNA gel electrophoresis and typical morphological changes observed under electron microscope proved the switch-on of apoptosis in terminally differentiating HL-60 cells.

Key words: $c$-myc oncogene, antisense transcripts, differentiation, apoptosis.

1. This work was supported by the National Medical and Scientific Key Item Grant

2. Corresponding author 


\section{INTRODUCTION}

The product of c-myc oncogene is a sort of oncoproteins that are closely related to malignant proliferation of tumor cells and as a transcriptional factor involved in the common pathway of proliferative signal transduction. It has been reported that overexpressed c-myc oncogene can promote cell growth in the presence of cytokines and induce apoptosis in the absence of cytokines[1,2]. The reason might include the expression of different genes promoted by c-myc oncogene expression under different circumstances. Therefore, apoptosis and proliferation might be two independent processes that have their own modulation system of signal transduction though the updated experimental data are still insufficient. Antisense c-myc oligodeoxynucleotides have been used to study the effects of the downregulated cmyc expression on differentiation[3] and apoptosis[4]. But what would happen in cell functional activities when c-myc oncogene expression decreased via transfection of recombinant plasmids expressing antisense c-myc transcripts? In the previous study, we investigated how cell proliferation was affected by the c-myc protein levels using antisense c-myc transcripts. In this article, inductions of terminal differentiation and apoptosis were observed.

\section{MATERIALS AND METHODS}

\section{Plasmids}

pUC18 was purchased from Promega. pE-H (containing 8.08kb human c-myc cDNA gene) and retroviral expression vector pDORneo were all kindly provided by Institute for Cancer Research, Chinese Academy of Medical Sciences.

\section{Construction of recombinant plsmids}

2723bp DNA fragment containing exon 1, intron 1 and 137nt exon 2 were obtained from PstIdigested pE-H plasmid. XbaI-digested $2723 \mathrm{bp}$ DNA fragment led to 5'-portion 1588bp fragment including exon 1 and a part of intron 1 and 3 '-portion 1135 bp fragment including a part of intron 1 and $137 \mathrm{nt}$ exon 2 . The 1135 bp fragment was ligated into the XbaI-Pst I sites of pUC18 according to the standard procedures[5].

\section{Cell culture and DNA transformation}

The HL-60 myeloid leukemia cell line was kindly provided by Lisheng Wang (Institute of Radiation Medicine, Beijing) and maintained in suspension cultures in RPMI 1640 supplemented with $10 \%$ heat-inactivated bovine serum at $37^{\circ} \mathrm{C}$ in a fully humidified atmosphere containing $5 \% \mathrm{CO}_{2}$. Cells in exponential growth were used in this study. The HL-60 cells were transfected with the plasmid pDORneo and pDACx by means of lipofection (lipofectin reagent from Life Sciences Technologies, Inc. USA) and the transfectants were named as DH and $\mathrm{CH}$ cells respectively. G418 (Life Sciences Technologies, Inc. USA) was added to culture $48 \mathrm{~h}$ later.

\section{Detection of c-myc protein}

Translation of c-myc mRNA into p65 was detected by cell-in situ ELISA. Cells were seeded in poly-L-lysine pretreated 96 -well plate at $4{ }^{\circ} \mathrm{C}$ overnight and fixed with $0.05 \%$ glutaraldehyde. After blocking, anti-p65 monoclonal antibody (C-33, Santa Cruz Biotechnology, USA) and HRPconjugated sheep-anti-mouse IgG were added sequentially. Then they were developed by adding 
TMB and $\mathrm{H}_{2} \mathrm{O}_{2}$. The reaction was stopped with $12.5 \%$ sulfuric acid. The value of OD492 was measured.

\section{Cytochemical stainings}

NBT (Nitroblue tetrazolium) reaction, peroxidase and $a$-NAE -naphthol acetate esterase) stainings were performed according to standard procedures[6]. The average positive rates of stainings were calculated through counting the positive and the total cell numbers of several vision fields under, microscope in three tests.

\section{Expressions of differentiation antigens CD13 and CD33[6]}

Cells $1.5 \times 10^{6}$ were mixed with 1:10 diluted anti-CD13 and anti- CD33 monoclonal antibodies (DAKO, Danmark) and reacted at $4^{\circ} \mathrm{C}$ for 40 minutes. After washing, 1:10 diluted FITCconjugated rabbit-anti-mouse IgG was added to $100 \mu$ l cell suspension and incubated in dark at 4 ${ }^{\circ} \mathrm{C}$ for 40 minutes. Cells were then analysed with FACS-440 (Becton Dickinson, USA).

\section{DNA fragmentation}

DNA was extracted from pDOR/HL-60 (DH) and pDACx/HL-60 $(\mathrm{CH})$ cells by salting-out method[7] after 5 to $6 \mathrm{~d}$ of transfection. DNA agarose gel electrophoresis was carried out as commonly described[5]. About $20 \mu \mathrm{g}$ DNA in $1 \times$ TBE buffered $1.3 \%$ agarose gel was electrophoresed at $35 \mathrm{~V}$ for about $10 \mathrm{~h}$.

\section{Morphology}

Ultrastructural features of HL-60 cells transfected and G418-treated were observed 5 to $12 \mathrm{~d}$ posttransfection under transmission electron microscope (TEM). In addition, the changes on the surfaces of cells in each group were also studied by scanning electron microscope (SEM).

\section{RESULTS}

\section{Construction of recombinant plasmids $p U C 18-P X$ and $p D A C x$}

Fig 1 outlined the construction of pDACx. Fig 2 shows the recovery of the useful c-myc fragments through restriction enzyme digestions. According to the physical map of $\mathrm{pE}-\mathrm{H}$, we used Pst I and Xba I to obtain the fragments of interest as described in MATERIALS and METHODS. In lane 2 of Fig 2, most of the 2723bp Pst I-fragments were cleaved by Xba I into 1588bp and 1135bp fragments, and the $1710 \mathrm{bp}$ and $1588 \mathrm{bp}$ were not separated in that electrophoresis. But what we are interested in is $1135 \mathrm{bp}$ fragment. The 1135bp (roughly $1.2 \mathrm{~kb}$ ) fragment was recovered from the gel and then ligated into pDORneo in antisense orientation called pDACx. The pUC18-PX and pDACx were further proved by restriction enzyme digestions, which is shown in Fig 3.

\section{Expression level of p65}

OD492 value of $\mathrm{DH}$ cells was $0.836+0.03$ while that of $\mathrm{CH}$ cells was $0.228+0.02$. The results indicated that the level of c-myc product decreased about 3.7 times at the 6th day posttransfection through expression of antisense transcripts to c-myc. 


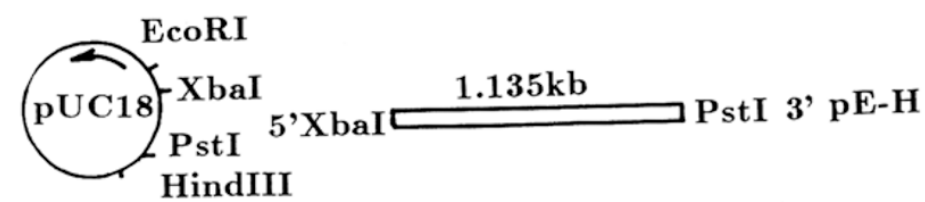

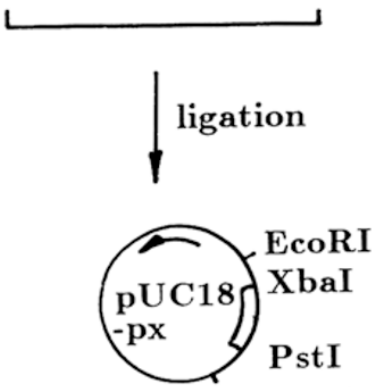

HindIII

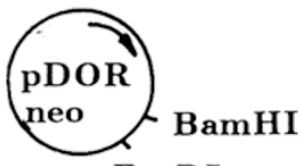

EcoRI

EcoRI-HindIII digestion
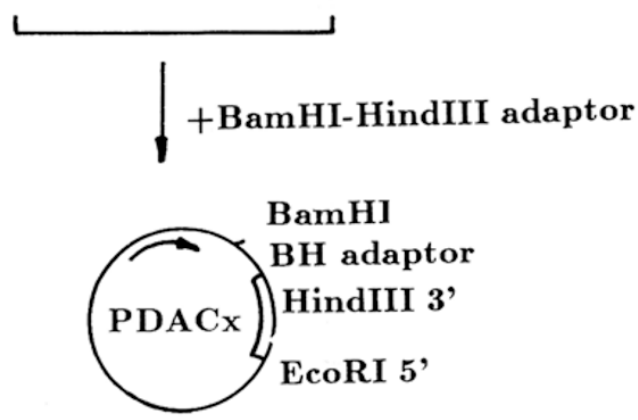

Fig 1. Diagram for pDACx constrcution

Decreased c-myc expression by antisense c-myc RNA accelerated myelomonocytic differentiation in $\mathrm{CH}$ cells

1. The occurrence of myelomonocytic differentiation in $\mathrm{CH}$ cells as revealed by the cytochemical staining of esterases and peroxidase

The average positive rate of NBT reduction of $\mathrm{CH}$ cells markedly increased at the 4 th day post transfection, from about $0.5-1.5 \%$ in $\mathrm{DH}$ cells to about $20-40 \%$ in $\mathrm{CH}$ cells. After the 6th day the positive rate began to decrease and gradually disappeared. The average positive rate of peroxidase-stained $\mathrm{CH}$ cells was $60-80 \%$ at the 5th day as compared with $10-20 \%$ in DH cells. Morever, the positive particles in $\mathrm{CH}$ cells were more and larger and those in $\mathrm{DH}$ cells were less and smaller. 
The peroxidase staining became negative till the 8th day. Positive results were also observed in $\mathrm{CH}$ cells with a-NAE staining.
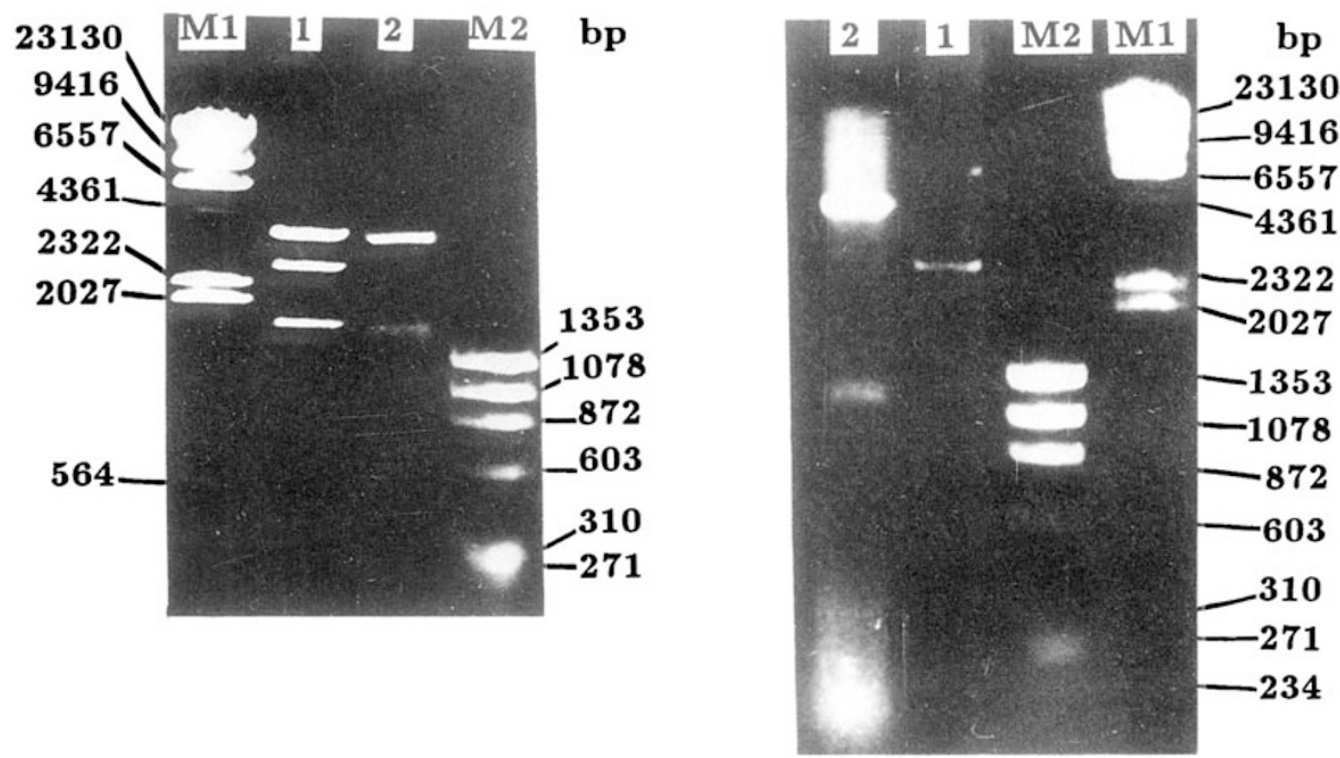

Fig 2. c-myc fragments were recovered from plasmids.

Lane 1: $\mathrm{pE}-\mathrm{H}$ digested with Pst I showing 3233, 2723, 1710 bp bands. Lane 2: pE-H digested with Pst I and Xba I showing 3233, 1710, 1588, 1135 bp bands.

DNA markers: M1, $\lambda$ DNA/Hind III; M2, $\varphi \chi$ 174RF DNA/Hae III.

Fig 3. Gel electrophoresis of the plasmids constructed after proper restriction enzyme digestions.

Lane 1: pUC18-PX was digested with Hind III and EcoRI, showing the 1182 inserted band and $2690 \mathrm{bp}$ vector band.

Lane 2: pDACx was digested with Hind III and EcoRI, showing about $1.2 \mathrm{~kb}$ exogenous fragment and 6.5 $\mathrm{kb}$ vector bands.

DNA markers: M1, $\varphi \lambda$ DNA/Hind III; M2, $\varphi \chi 174 \mathrm{RFDNA} / \mathrm{Hae}$ III.

2. The alterations of CD13 and CD33 antigen expression during myelomonocytic differentiation

Tab 1 shows the expression levels of CD13 and CD33 antigens in DH and CH cells. It could be seen that both the positive rate of CD13 antigen which defines myelomonocytic differentiation at the 4th day posttransfection and the average fluorescence intensity that indicates the density of CD13 antigen on each positive cell increased. Meanwhile, expression of CD33 antigen existing only in eariler myeloid 
progenitor cells decreased[8]. But on the 6th day posttransfection expression of CD13 antigen decreased and that of CD33 decreased further. The transiently increased CD13 expression and decreased CD33 expression in CH cells on the 4th day suggested that myelomonocytic differentiation could be induced in c-myc downregulated $\mathrm{CH}$ cells. The changes of differentiation antigen expression were in consistent with those of cytochemical stainings both in time and the results.

Tab 1. CD13 and CD33 antigen expression post transfection

\begin{tabular}{ccccc}
\hline cell & \multicolumn{2}{c}{ CD13 } & CD33 \\
\cline { 2 - 5 } type & positive rate(\%) & fluorescence intensity & positive rate(\%) & fluorescence intensity \\
\hline DH & $75.7 \pm 1.2$ & $48.8 \pm 2.1$ & $22.6 \pm 2.2$ & $38.8 \pm 2.4$ \\
CH(day 4) & $95.1 \pm 2.5$ & $71.4 \pm 3.3$ & $9.2 \pm 1.6$ & $40.2 \pm 2.2$ \\
CH(day 6) & $66.7 \pm 2.7$ & $54.6 \pm 2.5$ & $6.6 \pm 1.8$ & $42.4 \pm 2.9$ \\
\hline
\end{tabular}

\section{Apoptosis occurred following terminal differentiation in $\mathrm{CH}$ cells}

1. Nuclear DNA degradation

To seek for the reasons why increased CD13 expression soon went downwards on the 6th day after transfection, cell genomic DNA from DH and $\mathrm{CH}$ were analysed through agarose gel electrophoresis to examine the integrity of chromosome DNA. Fig 4 shows the results of DNA gel electrophoresis of $\mathrm{DH}$ and $\mathrm{CH}$ cells 5 days after transfection. DNA ladder bands with integer multiples of 180-200 base pairs were obviously seen in $\mathrm{CH}$ cells whereas no such ladder bands in DH cells.

Fig 4. DNA gel electrophoresis of DH and $\mathrm{CH}$ cells showing DNA ladder bands.

Lane 1: DH cells. Lane 2: $\mathrm{CH}$ cells (d 5). M: pGEM-3zf(+)/Hae III.

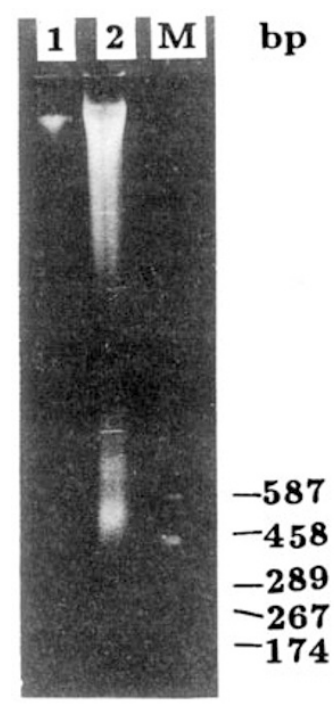


Hao XJ et al.

\section{Apoptotic morphological alterations}

Fig 5 shows the ultrastructural features of DH, G418-treated DH and $\mathrm{CH}$ cells observed under TEM. From the 5th day post transfection condensed heterochromatin in several crescent clumps that abutted against the nuclear envelope was observed in $\mathrm{CH}$ cells. The cytoplasmic membrane remained intact, organelles were atropied while enormous vacuoles and pultaceous bodies appeared in cytoplasm of the $\mathrm{CH}$ cells. The condensed nuclei were then further degraded, broken, moved out of the cells, and finally were encapsulated to form apoptotic bodies. This was consistent with the previous observations $[9,10]$. On the contrary, the morphological features of G418-treated DH cells were extreme dilation of endoplasmic reticula, destruction of mitochondria, collapse of cytoplasmic membrane and no obvious changes in chromatin and nuclear membrane (Fig 5A). The alterations in the surfaces of apoptotic cells under SEM have not been reported sofar (Fig 6). Enormous finger-like protuberances were seen on the surface of $\mathrm{DH}$ cells. However in that of $\mathrm{CH}$ cells the atrophy, smooth yet intact cytoplasmic membrane and sometimes the large concave regions, and clumpy projections were observed.

\section{DISCUSSION}

We transfected HL-60 cells with recombinant plasmids expressing antisense cmyc RNA to examine the effects of antisense c-myc transcripts on cell differentiation and survival. It has been reported that the c-myc oncogene was dominantly overexpressed in the poorly-differentiated cell lines, and contributed to the malignant proliferation and block of differentiation. It is commonly held decreased c-myc expression may cause differentiation. This study intended to add some answers to this question. Our experiments have shown that decrease of CD33, increase of CD13 expression, NBT reduction and functional activities of some enzymes such as $a$-naphthol acetate esterase and peroxidase were detected in $\mathrm{CH}$ cells, while no such alterations were observed in DH cells and HL-60 cells. There were no significant differences in morphology, enzymes activities and surface antigen expression between DH and HL-60 cells. It suggested that myelomonocytic differentiation occurred after p65 was downregulated through the expression of c-myc antisense transcripts. The amount of c-myc mRNA decreased during the HL-60 cells being induced to differentiate into granulocytes and monocyte-macrophages[11]. Terminal myelomonocytic differentiation was observed in this study along with the decreased c-myc expression. Thus the level of c-myc expression was closely correlated with myelomonocytic differentiation.

The result of DNA gel electrophoresis revealed non-random degradation of genomic DNA structure, which signified the destined death of these cells. The regular DNA ladder bands revealed in electrophoresis could provide the evidence for cell apoptosis. It was reported that this patterns of DNA ladder bands came from the specific cleavage of chromosome DNA at the linkage of internucleosomes due to the 
Promyelocytic leukemia cell line HL-60

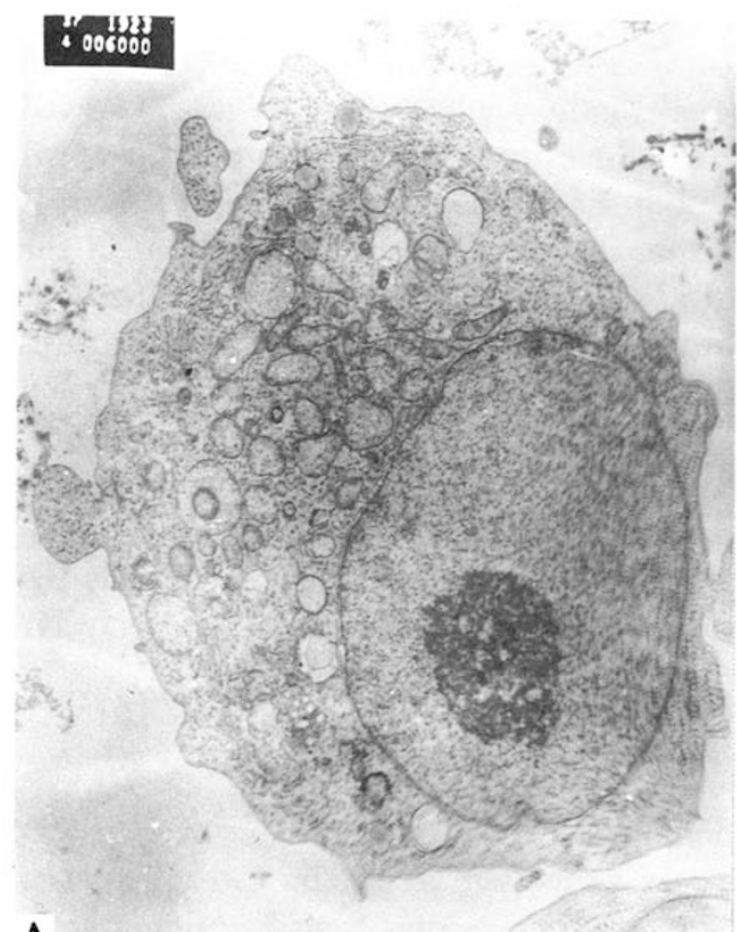

A

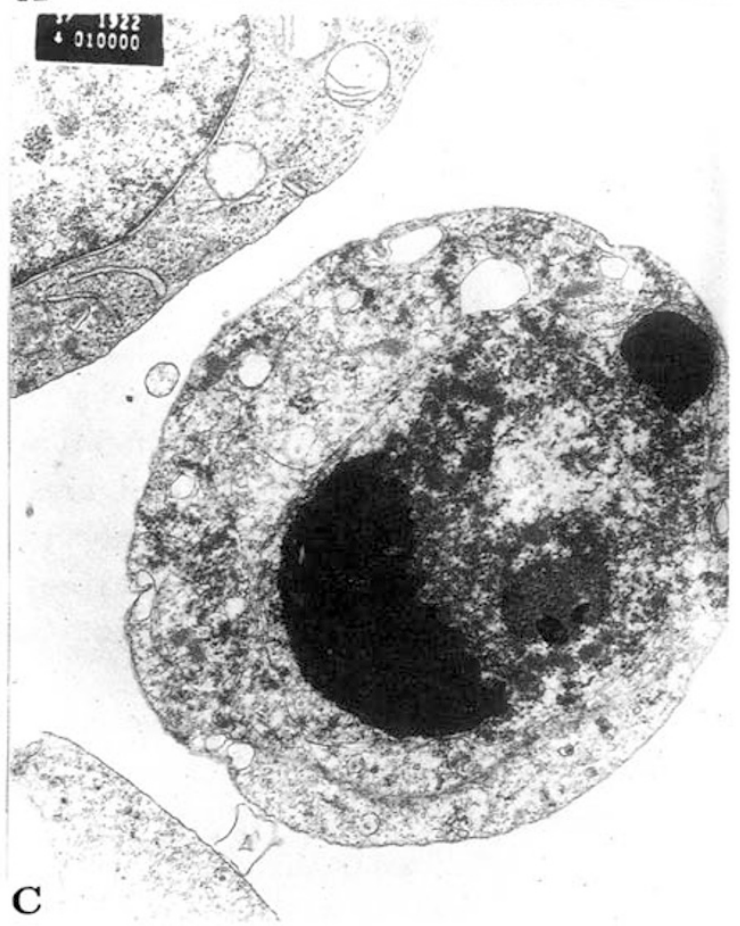

B
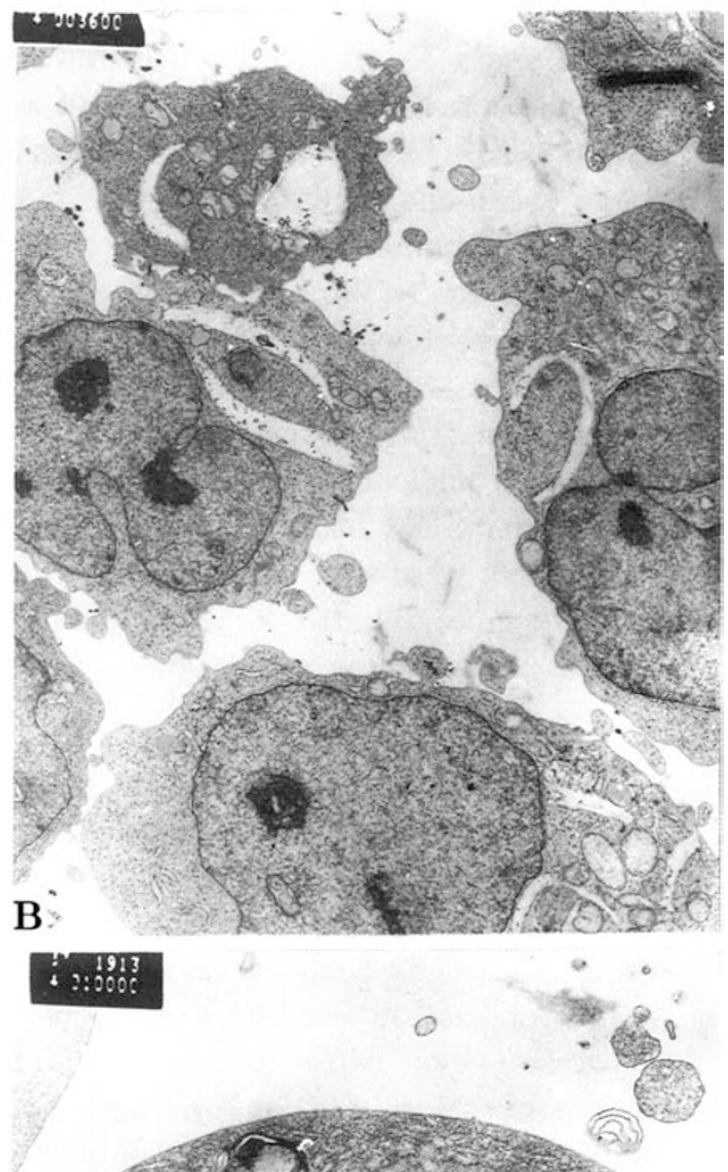

D 

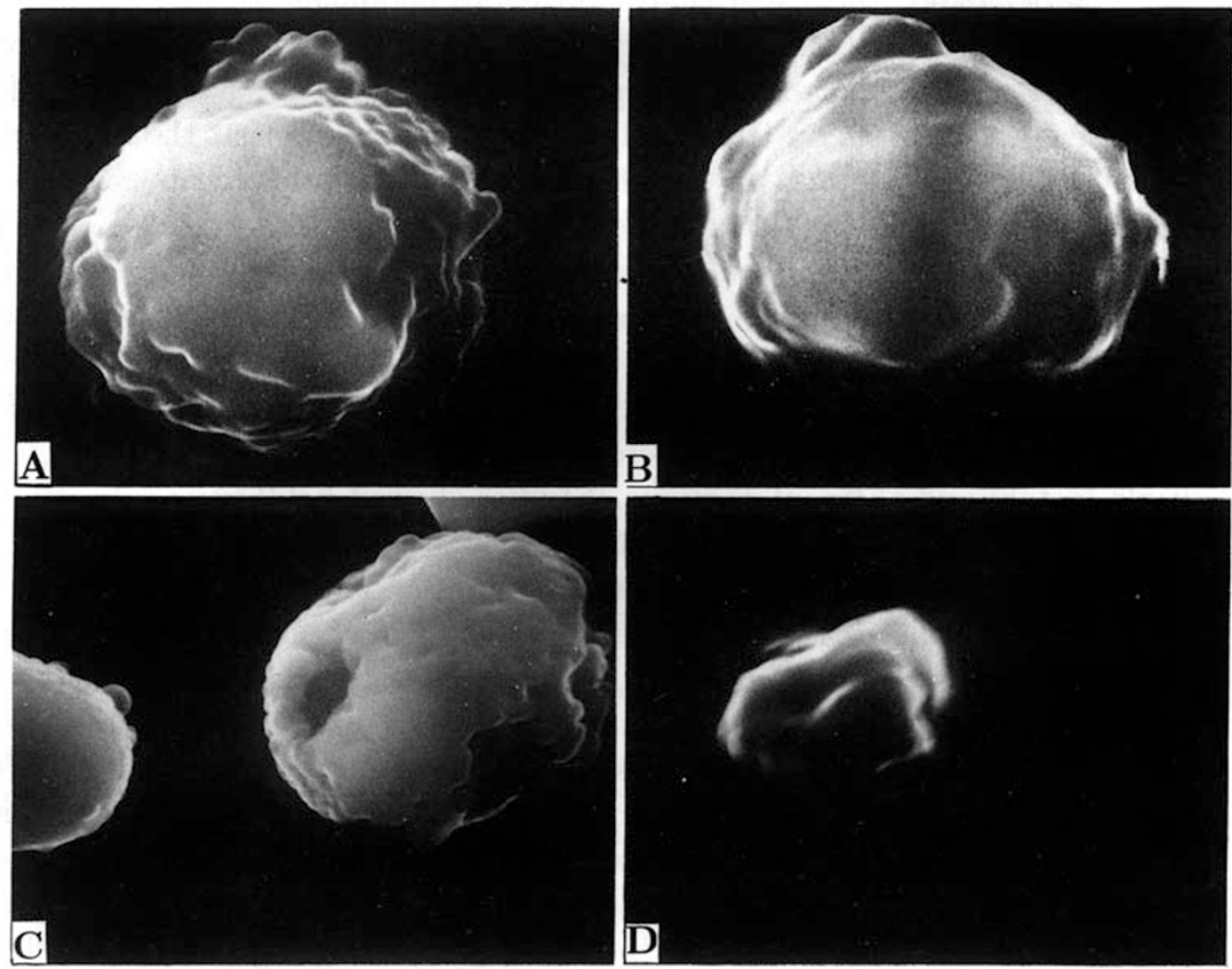

Fig 6. Surface alterations of $\mathrm{DH}$ and $\mathrm{CH}$ cells under SEM.

A: DH cells. Showing normal surface protuberances. $\times 5000$

B, C, D: CH cells. Showing clumpy, irregular projections $(B, \times 5000 ; \mathrm{D}, \times 10000)$ and large concave region $(\mathrm{C}, \times 3380)$.

$\triangleleft$ Fig 5. Ultrastructural features of $\mathrm{DH}$ and $\mathrm{CH}$ cells under TEM.

A: DH cell. Showing intact cytoplasmic and nuclear membranes, abundant cytoplasm and no changes in chromatin. $\times 6000$

B: G418/DH. Showing extreme dilation of organelles and destroyed mitochondria whereas no changes in chromatin. $\times 3600$

C, D: CH cells. Showing condensed heterochromatin arranged in sharply defined chromatin clumps that abutted against the dilated $(\mathrm{C}, \times 10000)$ and destroyed nuclear envelope, no obvious destruction in organelles and cytoplasmic membrane, some vacuoles and pultaceous bodies (arrow indicated) as well as nuclear pyknosis and fragmentation $(\mathrm{D}, \times 10000)$ 
activation of endonuclease in the early process of apoptosis[12]. The occurrence of DNA ladder bands suggested that endogenous endonuclease had been activated during apoptosis of $\mathrm{CH}$ cells. In morphological study, besides DH cells, we used G418-treated cells as controls to observe the morphological changes caused by cmyc antisense transcripts on HL-60 cells so as to emphasize that the morphological features of $\mathrm{CH}$ cells are unique, which is the characteristics of apoptosis but not necrosis as observed in G418-treated cells.

The relationship between the alteration in c-myc expression and differentiation and apoptosis has been studied previously[3, 13]. Although some investigators reported that the up-regulation of c-myc expression caused apoptosis[13] while others suggested that down-regulation may be necessary for the induction of apoptosis[14], both of them were correct under different circumstances. Apoptosis is the result of multiple factors and their interactions. Studies showed bcl-2 gene expression level decreased after differentiation of HL-60 cells[15]. With the deprivation of bcl-2inhibited apoptosis pathway, cells went to apoptosis. Therefore, differentiation was accompanied by apoptosis in HL-60 cells and bcl-2 may play an important role in this process. Yet bcl-2 itself did not have any effect on differentiation[16]. That the abrupt decrease of c-myc level in overexpressed HL-60 cells switched on the process of apoptosis was somewhat easy to understand due to the induction of differentiation. In addition, p53 may also be involved in this process. Lotem et al[1] reported that c-myc related apoptosis was not the direct result of deregulated c-myc expression but that c-myc expression increased cell's sensitivity to apoptosis and this sensitivity can be inhibited by the overexpressed mutant p53 and bcl-2 gene products.

From our results, it is concluded that in HL-60 cells transfected with c-myc antisense transcripts the following changes were observed: (i) the endogenous p65 expression was repressed; (ii) the decrease in p65 resulted in the increased expression of the myelomonocytic differentiating cell surface marker CD13, increased NBT reduction, positive peroxidase and $a-\mathrm{NAE}$ stainings; (iii) DNA degradation and typical morphological changes suggesting apoptosis. Therefore, it appears that the decreased c-myc expression might be one of the causes of induced terminal differentiation and apoptosis in HL-60 cells.

\section{ACKNOWLEGEMENTS}

We thank Mr. Zhang Lianfeng, Wang Zhihua and Wang Xiuqin from Institute of Cancer Research, Chinese Academy of Medical Sciences for helping in plasmid construction.

\section{REFERENCES}

[1] Lotem J, Sachs L. Regulation by bcl-2, c-myc and p53 of susceptibility to induction of apoptosis by heat shock and cancer chemotherapy compounds in differentiation competent and defective 
myeloid leukemia cells. Cell Growth Differ 1993; 4:41-9

[2] Sachs L, Lotem J. Control of programmed cell death in normal and leukemia cells: new applications for therapy. Blood 1993; 82:15-21

[3] Holt JT, Rendner RL and Nienhuis AW. An oligomer complementary to c-myc mRNA inhibits proliferation of HL-60 promyelocytic cells and induces differentiation. Mol Cell Biol 1988; 8:96373

[4] Kimura S, Maekawa T, Hirakawa K, et al. Alterations of c-myc expression by antisense oligodeoxy nucleotides enhanced the induction of apoptosis in HL-60 cells. Cancer Res 1995; 55:1379-84

[5] Sambrook J, Pritsch EF, Maniatis T. Molecular Cloning: A Laboratory Manual. 2nd ed. New York: Cold Spring Harbor Laboratory Press. 1989

[6] Kamano H, Ohnishi H, Tanaka T, et al. Effects of the antisense v-myb expression on K562 human leukemia cell proliferation and differentiation. Leuk Res 1990; 14:831-9

[7] Miller SA, Dykes DD, Polesky HF. A simple salting out procedure for extracting DNA from human nucleated cells. Nucleic Acids Res 1988; 16:1215-8

[8] Andrews RG, Singer JW and Bernstein ID. Precursors of colony-forming cells in humans can be distinguished from colony-forming cells by expression of the CD33 and CD34 antigens and light scatter properties. J Exp Med 1989; 169:1721-31.

[9] Ormerod MG. Flow cytometric studies of apoptosis. CMB 1994; 1:35-43

[10] Bergamashi G, Rosti V, Danova M, et al. Apoptosis: biological and clinical aspects. Haematologica 1994; 79:86-93

[11] Koeffer HP. Induction of differentiation of human acute myelogenous leukemia cells: therapeutic implications. Blood 1983; 62:709-21.

[12] Fernandes RS and Cotter TG. Activation of a calcium magnesium independent endonuclease in human leukemia cell apoptosis. Anticancer Res 1993; 13:1253-60

[13] Shi Y, Glynn JM, Guilbert LJ, et al. Role for c-myc in activation-induced apoptotic cell death in T cell hybridomas. Science 1992; 257:212-4

[14] Alnemri ES, Fernandes TF, Haldar S, et al. Involvement of BCL-2 in glucocorticoid-induced apoptosis of human pre-B-leukemias. Cancer Res 1992; 52:491-8

[15] Delia D, Aliello A, Soligo D, et al. Bcl-2 proto-oncogene expression in normal and neoplasic human myeloid cells. Blood 1992; 79:1291-8

[16] Naumovski L, Cleary ML. Bcl-2 inhibits apoptosis associated with terminal differentiation of HL-60 myeloid leukemia cells. Blood 1994; 83:2261-7

Received 19-12-1995. Revised 17-12-1996. Accepted 23-12-1996. 